\title{
Gestión de la información para la logística de operaciones offshore en Colombia
}

\section{Information management for the logistic operations offshore in Colombia}

\author{
págs. $40-52$ \\ Grupo de Investigación: CINDE \\ Línea de Investigación: Logística \\ Mónica Yinette Suárez Serrano*, Esteban Felipe González Amado•• \\ \& Johandra Liseth González Ramírez...
}

Recibido: 28 de enero de 2016 Aceptado: 21 de marzo de 2016

\section{Resumen}

En los procesos de perforación offshore, es necesario tener sistemas que garanticen el flujo de información, debido a que las fallas logísticas derivadas de errores en la comunicación pueden aumentar los costos y los tiempos de operación. En este sentido, el objetivo principal de esta investigación es diseñar un sistema de gestión de la información para el apoyo logístico de la perforación offshore, que facilite el abastecimiento de forma óptima y oportuna de los requerimientos de las plataformas ubicadas mar a dentro. La construcción del sistema se basó en la identificación de las causas de fallas de comunicación, a través de la revisión de fuentes secundarias, las cuales fueron validadas posteriormente con el uso del método Delphi.

Palabras claves: Gestión de la información, Logística y offshore.

\begin{abstract}
In offshore drilling process, it is necessary have systems to ensure the information flow, because the logistical failures resulting from miscommunication can increase costs and time in operation. The objective of this research is to design a system of information management for logistical support of offshore drilling to facilitate optimal supply and timely requirements of sea platforms located inside. The construction of the system was based on the identification of causes of failures of communication, through the review of secondary sources, which were subsequently validated using the Delphi method.
\end{abstract}

Keywords: information management, logistic and offshore.

\footnotetext{
- Ingeniera Industrial, Especialista en Gestión en Redes de Valor y Logística, Docente Investigadora. Universidad de América, monica.suarez@investigadores.edu.co.

•• Ingeniero Industrial, Coinvestigador, Universidad de América, estebanfelipe1991@hotmail.com.

... Ingeniero Industrial, Coinvestigador, Universidad de América, johandragonzalezr@gmail.com.
} 


\section{Introducción}

Los sistemas de gestión de información apoyan las operaciones logísticas de perforación offshore, al permitir que la información fluya de un punto a otro por los canales y nodos correctos, manteniendo su estructura original, objetividad y garantizando parámetros de seguridad, actualización, registro y almacenamiento; mediante conceptos de integración, usabilidad y confidencialidad.

Teniendo en cuenta los resultados obtenidos de la revisión de fuentes primarias y secundarias que se precisaran a lo largo del documento, se evidencia la existencia de fallas en los flujos de información en las actividades logísticas de las operaciones offshore, por lo cual esta investigación pretende contribuir a minimizarlas a través de la propuesta de un sistema que incluye el diseño flujos correctos y una herramienta computacional que apoye su aplicación.

\section{Método}

El sistema de información se diseñó en dos fases: una fase de diagnóstico y una de diseño. La primera de ellas, consistió en identificar las causas asociadas a las fallas en los flujos de información en las operaciones logísticas que apoyan los procesos de perforación offshore, las cuales fueron definidas inicialmente, a través de la revisión de fuentes secundarias y posteriormente validas a través de la aplicación del método Delphi, en el cual se incluyó la participación de expertos en perforación a nivel nacional e internacional.

Para apoyar el proceso de validación de las causas en el marco del desarrollo del método Delphi se aplicó una entrevista estructurada y dirigida a los expertos, la cual se construyó bajó el apoyo de los principios de la Psicometría TRI (Teoría de Respuesta al Ítem) y las escalas de Likert.

Una vez identificadas las causas y priorizadas con el uso de la Matriz Vester, se procede a caracterizar los procesos del área logística para operaciones off-shore basados en las en la cadena de valor propuesta por Ronald $\mathrm{H}$. Ballou en su libro Administración de la Cadena de Suministro (2014) y la propuesta por Michael Porter en Competitive advantage: creating and sustaining superior performance (1998); para finalmente definir la asociación de las causas con cada uno de los procesos del área logística para operaciones off-shore establecidas en el diseño de la red de valor.

La fase de diseño, inició con una función exploratoria de fuentes secundarias para realizar un análisis comparativo de los modelos, mecanismos, procesos y herramientas de gestión de información adecuado para el funcionamiento óptimo de la actividad logística para las operaciones off-shore en Colombia. Los resultados de este análisis permitieron identificar las características principales del sistema de información propuesto.

Dado lo anterior se procedió a caracterizar y segmentar la Cadena de suministro e identificar las necesidades de información en el área logística para operaciones offshore, para finalmente construir la estructura del sistema, el flujo de información y la herramienta computación en Microsoft Access.

\section{Resultados}

A continuación, se presentan los resultados obtenidos en cada una de las fases descritas en el método.

\subsection{Diagnóstico}

En consecuencia, con lo descrito en el método, a continuación, se presentan los resultados obtenidos en cada una de las etapas del diagnóstico.

Identificación de causas-revisión fuentes secundarias. Como resultado de la revisión de las fuentes secundarias se construyó un diagrama Ishikawa mixto que integró las metodologías 
de las $6 \mathrm{M}$ y de estratificación (Gutiérrez y De la Vara, 2013), definiendo como causas principales las siguientes: Métodos de trabajo, Medición, Maquinaria (ERP), Materiales (información), Medio Ambiente, Mano de obra y Marco Legal, como se muestra en la Figura 1.

- Métodos de trabajo. En este punto se determinan las causas consideradas influyentes en las fallas en el flujo de información que se relacionan con las formas de llevar a cabo las operaciones, los parámetros, directrices y modelos. Para ello, se analizaron las actividades logísticas como apoyo a actividades de perforación de pozos para la extracción de hidrocarburos en ambientes costa afuera, en consecuencia las causa secundarias asociadas a los métodos de trabajo son: modelos de suministro improvisados, canales de transmisión de información inadecuados, inexistencia de clasificación de suministros, integración deficiente en los sistemas de información existentes, procesos extensos en fases de solicitud-aprobación, aprobaciónadquisición, adquisición-despacho, inexistencia de filtros de comprobación de información para evitar errores humanos, falta de retroalimentación de la información, fallas en la gestión de la cadena de suministro, ausencia en la definición de responsables y usuarios de la información y múltiples medios para la transmisión de información (Karalkova y Kilcheuskaya, 2011; Favilla, Claessens, Mello y Flach, 2012; Chima, 2007).

- Medición. Los flujos de información pueden verse afectados por variaciones y cambios entre variables de uso y el seguimiento de materiales e información cuando no se tiene un correcto estándar para delimitar valores permisibles o normales. El no seguir el comportamiento de los datos puede afectar que la información disponible no sea correcta o transmitida en tiempo real, conllevando a fallos en los nodos que utilizan dicho recurso y por ende afectando las operaciones logísticas y de perforación en general.
Los controles periódicos y evaluación continua de equipos tanto a nivel físico como operacional, pueden mitigar la ocurrencia de las fallas en las actividades logísticas que apoyan la perforación mar a dentro. Las subcausas relacionadas con la medición son: cadena de suministro indefinida, planeación de recursos inadecuada, seguimiento limitado a la calidad de los datos, calibración deficiente en equipos usados en metoceánica, cuantificación inadecuada de recursos para plataforma offshore y control limitado al volumen de datos (Karalkova et al., 2012; Fontana, Papa, Marte, Yoshioka y Sakurai, 2014).

- Maquinaría (ERP). Para la presente investigación, la Maquinaria (ERP) son los mecanismos empleados para llevar a cabo los flujos de información desde su punto de origen a su punto de destino. También se incluyen medios físicos de comunicación, modelos informáticos y mecanismos para el registro. Las subcausas que hacen parte de esta causa principal, son:

Medios de comunicación y transmisión de información inadecuados, ausencia de plataformas y sistemas de registro de información, utilización deficiente de software y hardware para la gestión de la información e incompatibilidad en los sistemas gestión de información (Fontana et al., 2014; Chima, 2007).

- Materiales (Información). El "Material” en el análisis del problema identificado es la información, la cual es considerada como factor clave para el cumplimiento óptimo de operaciones logísticas y de perforación. Dentro de estos aspectos, deben incluirse elementos de aseguramiento y registro que permitan su disponibilidad continua y en tiempo real. En el análisis de esta causa se identificaron como subcausas:

Falta de disponibilidad de registros apropiados de la operación, no existe claridad a cerca de qué información se debe compartir y desviaciones en la información compartida 
(Karalkova y Kilcheuskaya, 2011; Fontana et al., 2014).

- Medio ambiente. Involucra aspectos que puedan influir de forma directa e indirecta en el entorno en el que se desarrollan las operaciones logísticas offshore y que pueden provocar interrupción o fallas en los flujos de información. El conjunto de subcausas son:

Emisiones por subutilización de la capacidad de modos de transporte, ausencia de registros sobre condiciones meteorológicas, suministro de requerimientos sin identificación ni etiquetas y limitaciones de acceso a la información geográfica (Kukareko y Kiril, 2013).

- Mano de obra. Incluye aspectos relacionados con el personal, sus conocimientos, habilidades, destrezas, y cualidades que influyen sobre la operación logística. En una adecuada gestión de la información, es importante que las personas tengan conocimientos sobre las directrices laborales, la cadena de mando, los protocolos y procesos que se deben cumplir. Las subcausas asociadas a la "Mano de Obra" son las siguientes:

Falta de capacitación, transmisión de información interpersonal e interdepartamental de mala calidad, inoportuna e incompleta, resolución de problemas a partir de la intuición e improvisación sin herramientas de calidad, personal calificado limitado, desconocimiento general de las directrices de los proyectos y desconocimiento de la cadena de mando (Karalkova y Kilcheuskaya, 2011; Fontana et al., 2014; Favilla et al., 2012).

- Marco legal. La normatividad legal es primordial en la garantía de un flujo óptimo de la información pues del conocimiento legal y el cumplimiento de las normas depende el curso de las operaciones. La importancia recae en la naturaleza de la industria, ya que, debido a los impactos y riesgos generados por la actividad, las empresas pueden incurrir en acciones inaceptables que generen sancio- nes, penalizaciones o ceses operacionales. Conocer la ley permite que la información que fluya en relación con éstos términos sea veraz y confiable, disminuyendo la posibilidad de costosos errores y otras afectaciones operativas.

En algunos casos la falta de claridad en la legislación laboral vigente, en la normatividad de perforación offshore y de los lineamientos legales para el transporte de personas, alimentos, equipos y materiales, al igual que la falta de orientación en los límites marítimos legales, son causas de las fallas en la información de las operaciones de perforación (Rajoo, 2006; Fontana et al., 2014).

Priorización de causas. Organizadas las causas en el diagrama de Ishikawa, se priorizaron según la incidencia en las fallas en el flujo de la información. Para esto se utilizó la Matriz Vester que permitió clasificarlas en críticas, activas, pasivas e indiferentes, a partir de una valoración con puntajes de 0 a 3, siendo 0 nada incidente y 3 muy incidente. Es de resaltar que esta valoración se hace en relación a la influencia que tiene una causa sobre las demás y las demás sobre ésta. Como resultado de la priorización se obtienen 12 causas críticas, 7 activas, 12 pasivas y 7 indiferentes, la cuales se especifican en la Tabla 1.

Caracterización de la cadena de valor del área logística para operaciones off-shore. Como parte del diagnóstico, es importante identificar las actividades que intervienen en el intercambio de información dentro del área logística, ya que gracias a éstas, se identifican características de los recursos utilizados y el significado que adquiere la información en las actividades relacionadas con la satisfacción del cliente final, en este caso, la plataforma de perforación.

Ronald $\mathrm{H}$. Ballou en su libro Administración de la Cadena de Suministro (2004), nos permite realizar el primer acercamiento a la caracterización de la cadena de valor a través de la 
clasificación de las actividades que se dirigen para conformar la logística, actividades centrales y de apoyo.

El segundo referente a considerar será Michael Porter con su cadena de valor propuesta. Porter define la cadena de valor como una herramienta de gestión que permite realizar un análisis interno a una organización a través de su desagregación en las principales actividades generadoras de valor (Elias, 2014).

Con base en la caracterización de Porter y Balluo se definen las actividades que hacen parte de la cadena de valor para el abastecimiento de las operaciones offshore y se relacionaron con las causas identificadas anteriormente. Los resultados se muestran en la Figura 2 y en la Tabla 2.

Tabla 1.

Resultados de la priorización

Pasivos

Canales de transmisión de información inadecuados

Desconocimiento de la legislación de transporte de personas, alimentos, equipos y materiales

Ausencia de plataformas y sistemas de registro de información Cadena de suministro indefinida

Desconocimiento de la legislación para perforación offshore Medios de comunicación y transmisión de información inadecuados Incompatibilidad en los sistemas de gestión de información Ausencia en la definición de responsables y usuarios de la información

Múltiples medios para la transmisión de la información

Desconocimiento general de las directrices de los proyectos Falta de conocimiento sobre los límites marítimos legales Falta de capacitación

\section{Críticos}

Uso deficiente de estrategias y conceptos ERP para la perforación

Utilización deficiente de software y hardware para la gestión de la información

Modelos de suministro improvisados

Integración deficiente de los sistemas de información existentes

Desconocimiento de la cadena de mando

Fallas en la gestión de la cadena de suministro

Seguimiento limitado a la calidad de los datos

Falta de registros apropiados

Control limitado al volumen de datos e información

Falta de retroalimentación de la información

Planeación de recursos inadecuada

No existe claridad acerca de que información se debe compartir

\begin{tabular}{|c|c|}
\hline Indiferentes & Activos \\
\hline Inexistencia de clasificación de suministros & Mala calidad en la información a compartir \\
\hline $\begin{array}{l}\text { Inexistencia de filtros de comprobación de informa- } \\
\text { ción para evitar errores humanos }\end{array}$ & $\begin{array}{l}\text { Cuantificación inadecuada de recursos para plataforma } \\
\text { offshore }\end{array}$ \\
\hline Limitaciones de acceso a la información geográfica & $\begin{array}{l}\text { Transmisión de información interpersonal e interdepar- } \\
\text { tamental de mala calidad, inoportuna e incompleta }\end{array}$ \\
\hline Personal calificado limitado & $\begin{array}{l}\text { Procesos extensos en fases de solicitud-aprobación, } \\
\text { aprobación-adquisición, adquisición-despacho. }\end{array}$ \\
\hline $\begin{array}{l}\text { Suministro de requerimientos sin identificación ni } \\
\text { etiquetas }\end{array}$ & Emisiones por subutilización de recursos de transporte \\
\hline $\begin{array}{l}\text { Calibración deficiente en equipos usados en meto- } \\
\text { ceánica. }\end{array}$ & $\begin{array}{l}\text { Resolución de problemas a partir de la intuición e im- } \\
\text { provisación sin herramientas de calidad }\end{array}$ \\
\hline Desconocimiento de la legislación laboral vigente & Ausencia de registros sobre condiciones meteorológicas \\
\hline
\end{tabular}

Fuente: elaboración propia. 
Figura 1.

Diagrama Ishikawa. Causas identificadas a través de fuentes secundarias

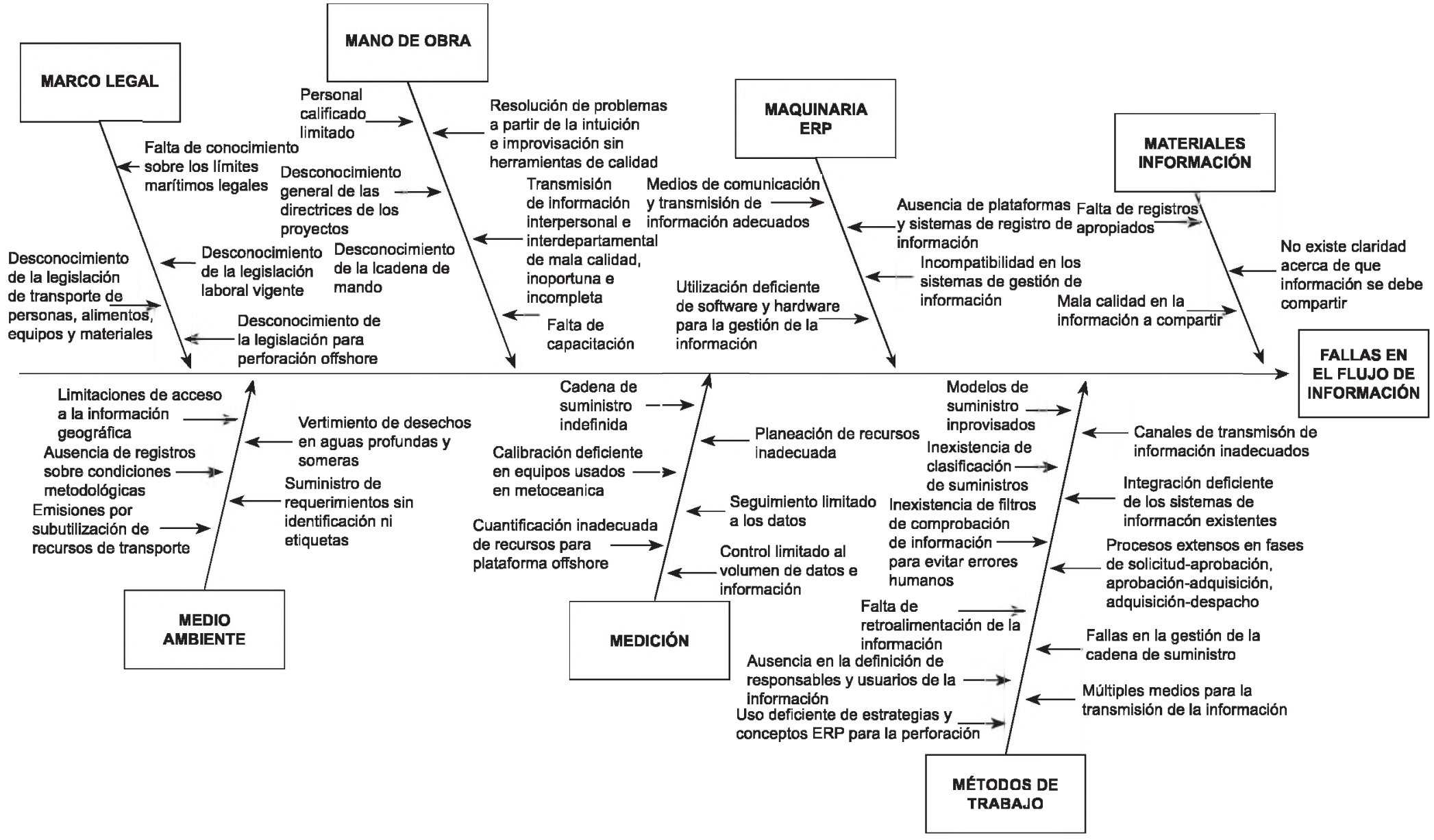

Fuente: elaboración propia. 
Validación de causas. Como se mencionó anteriormente la validación de las causas identificadas a través de fuentes secundarias, se realizó con la aplicación del método Delphi y con él una aplicación de entrevistas estructuras y dirigidas a seis expertos contactados con ayuda de la técnica Bola de Nieve. La metodología empleada permitió analizar experiencias en el área logística de proyectos onshore y offshore realizados en Colombia; en el Golfo de México y en el Mar del Norte. Dentro de las experiencias analizadas para Colombia cabe destacar la experiencia de los expertos en el desarrollo del proyecto Mapalé en la Costa Caribe Colombiana.

Como resultado del proceso de validación se definieron como causas que harán parte constitutiva del sistema de información, las siguientes: procesos extensos en fases de solicitud-aprobación, aprobación-adquisición, adquisición-despacho, planeación de recursos inadecuada, disponibilidad de las referencias en el stock del proveedor, transmisión de información interpersonal e interdepartamental de mala calidad, inoportuna e incompleta, desconocimiento de la legislación de transporte de personas, alimentos, equipos y materiales, canales de transmisión de información inadecuados, integración deficiente de los sistemas de información, ausencia en la definición de responsables y usuarios de la información, múltiples medios para la transmisión de la información, medios de comunicación y transmisión de infor- mación inadecuados, ausencia de plataformas y sistemas de registro de información, no existe claridad acerca de qué información se debe compartir, mala calidad en la información a compartir, desconocimiento de los límites marítimos legales, falta de conocimiento sobre tratados marítimos internacionales, desconocimiento de estándares mundiales de transmisión de información, políticas de comercio exterior insuficientes, desconocimientos de la reglamentación ambiental, inexistencia de filtros de comprobación de información para evitar errores humanos, uso deficiente de estrategias y conceptos ERP para la perforación, falta de capacitación, personal calificado limitado, desconocimiento general de las directrices de los proyectos, desconocimiento de la cadena de mando, inexistencia de clasificación de suministros, suministro de requerimientos sin identificación ni etiquetas, cuantificación inadecuada de recursos para plataforma offshore, falta de registros apropiados, emisiones por subutilización de recursos de transporte, ausencia de estandarización para el vertimiento de desechos en aguas profundas y someras, falta de estándares en el desarrollo de planes de contingencia ante contaminación del ecosistema marino, utilización deficiente de software y hardware para la gestión de la información, incompatibilidad en los sistemas de gestión de información, modelos de suministro improvisados, resolución de problemas a partir de la intuición e improvisación sin herramientas de calidad y cadena de suministro indefinida.

Figura 2.

Cadena de valor de abastecimiento para operaciones off-shore.

\begin{tabular}{|c|c|c|}
\hline \multicolumn{2}{|c|}{ Gestión de seguridad de la información } \\
\hline & Gestión de recursos humanos \\
\hline & Gestión para transmisión de información \\
\hline & Gestión de registros de información \\
\hline Gestión de procedimientos \\
\hline $\begin{array}{c}\text { Gestión de } \\
\text { requerimientos }\end{array}$ & $\begin{array}{c}\text { Gestión de riesgos y programas de contingencia } \\
\text { contrataciones/ } \\
\text { proveedores }\end{array}$ & $\begin{array}{c}\text { Gestión de } \\
\text { inventarios }\end{array}$ \\
\hline
\end{tabular}

Fuente: elaboración propia. 
LÍNEA DE INVESTIGACIÓN: LOGÍSTICA

Tabla 2.

Causas asociadas a las actividades de la cadena de valor.

\begin{tabular}{|c|c|}
\hline Actividad & Causas \\
\hline Gestión de requerimientos & $\begin{array}{l}\text { Cuantificación inadecuada de recursos para plataforma offshore. } \\
\text { Cadena de suministro indefinida. }\end{array}$ \\
\hline Gestión de inventarios & $\begin{array}{l}\text { Inexistencia de clasificación de suministros. } \\
\text { Suministro de requerimientos sin identificación ni etiquetas. }\end{array}$ \\
\hline Gestión de abastecimiento & $\begin{array}{l}\text { Emisiones por subutilización de recursos de transporte. } \\
\text { Modelos de suministro improvisados. } \\
\text { Planeación de recursos inadecuada. }\end{array}$ \\
\hline $\begin{array}{l}\text { Gestión de seguridad de la } \\
\text { información }\end{array}$ & $\begin{array}{l}\text { Inexistencia de filtros de comprobación de información para evitar errores hu- } \\
\text { manos. }\end{array}$ \\
\hline Gestión de recursos & Desconocimiento de la cadena de mando. \\
\hline humanos & $\begin{array}{l}\text { Ausencia en la definición de responsables y usuarios de la información. } \\
\text { Falta de capacitación. } \\
\text { Personal calificado limitado. } \\
\text { Desconocimiento general de las directrices de los proyectos. } \\
\text { Falta de conocimiento sobre los límites marítimos legales. } \\
\text { Desconocimiento de la legislación laboral vigente. } \\
\text { Desconocimiento de la legislación de transporte de personas, alimentos, equi- } \\
\text { pos y materiales. } \\
\text { Desconocimiento de la legislación para perforación offshore. }\end{array}$ \\
\hline
\end{tabular}

\subsection{Diseño del sistema de información}

Es importante mencionar que el sistema de información se encuentra diseñado para las operaciones dadas entre el shore-base y la plataforma de perforación (segmento definido de la Cadena de suministro de gas y petróleo).

Una vez definido el segmento de la Cadena de suministro para el cual será aplicable el sistema de información, se procede también a establecer a partir del análisis de las fuentes primarias consultadas (entrevistas), los requerimientos de información en las operaciones logísticas que apoyan la perforación costa afuera. La articulación de cada uno de los resultados parciales de la investigación citados anteriormente, dan lugar a definir el sistema de información, el cual se diseñó para integrar las áreas que representan a uno o varios de los actores de la cadena de suministro (proveedor, base de operaciones, buques y helicópteros y plataforma).

Como se muestra en la Figura 3. el sistema de información se representa por el círculo central y las líneas verdes de doble sentido representan el acceso a las áreas de materiales, base de suministro, recursos humanos, proveedores y plataforma con círculos de rosado, morado, naranja, verde y azul oscuro respectivamente. Las líneas delgadas, también de doble sentido conexas a cada una de las áreas, representan el flujo de información constante con el que deben contar las áreas para asegurar el éxito de la operación de abastecimiento.

En un segundo nivel encontramos círculos más pequeños de color azul claro, que acompañan a cada una de las áreas, estos círculos representan las actividades primarias y dos de las actividades secundarias de la cadena de valor; 
estas actividades incluyen las gestiones de requerimientos, contratación y proveedores, inventarios y abastecimiento consideradas primarias y las gestiones de recursos humanos, riesgos y programas de contingencia consideradas secundarias o de apoyo.

En un último nivel se puede observar tres grandes círculos en color gris claro que abarcan a todas las áreas y sus sub-proceso, estos circulos simbolizan las cuatro actividades secundarias restantes; las gestiones de procedimientos y registros, transmisión y seguridad de la información.

Como parte del sistema se definen también los flujos de información desde el momento que se identifica una necesidad en la plataforma de perforación hasta el instante en que se satisface. El proceso inicia en el pozo ubicado en la plataforma de perforación costa afuera en la que el company man o su asistente se encarga de realizar el proceso de requerimiento, de material o de personal, al área de compras o recursos humanos, respectivamente. Si la necesidad coresponde a personal esta se transmite al encargado de recursos humanos quien realiza una solicitud que responda a las caracteristicas especificadas por el company man o su asistente. Posteriormente se lleva a cabo el proceso de reclutamiento y selección que de ser exitoso termina en la contratación y la llegada del personal a la plataforma; si por el contrario, la necesidad corresponde a material, el área de compras recibe el requerimiento generando una solicitud de pedido que puede resultar en dos casos. El primero, cuando el material ya se encuentra ubicado en la base de suministro y solo debe seguir un proceso de envio; o el segundo, cuando el material debe ser adquirido a través de un proveedor, originando la creación de una orden de compra.

Figura 3.

Diseño del sistema de gestion de información.

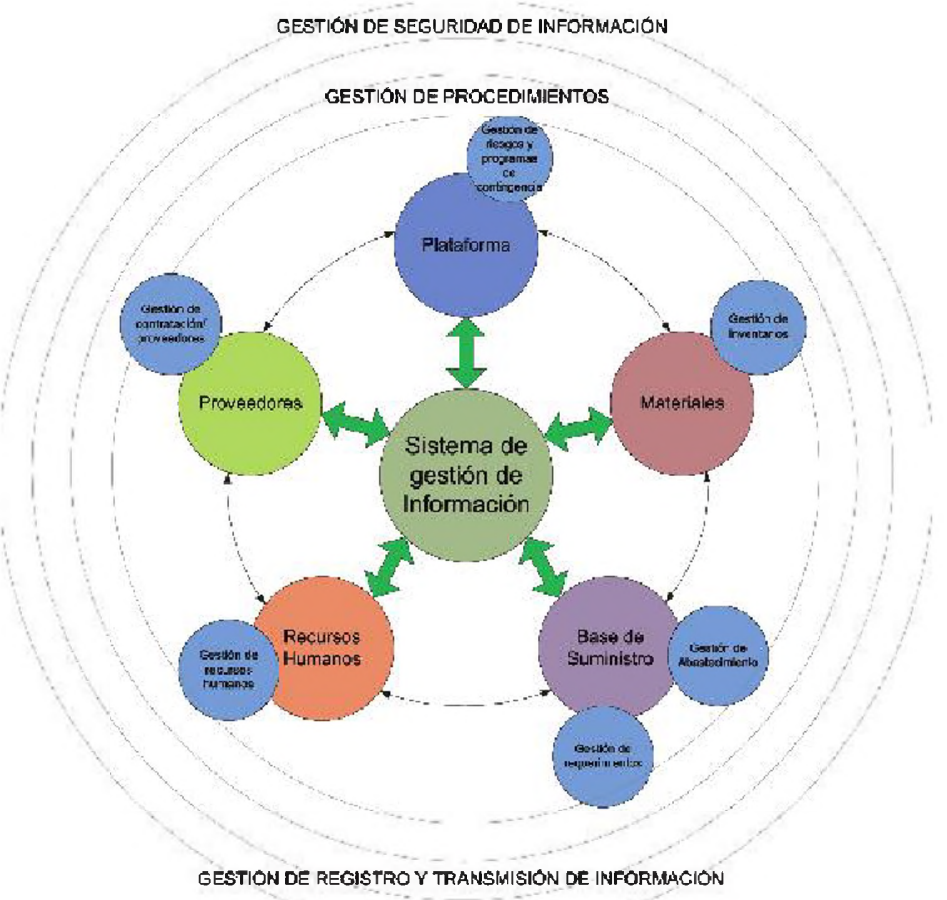

Fuente: elaboración propia. 
La secuencia del primer caso inicia con la generación de una orden de envío, la cual entra en marcha en el momento que el medio de transporte (buque o helicóptero) llega a la base de suministro para que le sea asignado un muelle y posterior al proceso de descarga del material que retoma de la plataforma, se carga el material relacionado en la orden de envio, se realiza el despacho del modo de transporte y finalmente se entrega el material en la base de operaciones.

Por otro lado, la secuencia del segundo caso, inicia con la selección del proveedor que mejor se ajuste a las necesidades descritas en el requerimiento realizado desde la plataforma para posteriormente generar una orden de compra que se da por terminada en el momento de recepción en la base de suministro, seguida del proceso de descarga, en este momento se debe decidir si la necesidad es de inmediato cumplimiento, caso en el que se realiza, en seguida, una orden de envío; o si por el contrario es material para envios futuros, éste se debe almacenar. El flujo descrito anteriormente se puede observar en las Figuras 4 y 5.

Figura 4.

Diagrama de flujo Sistema de Gestión de información

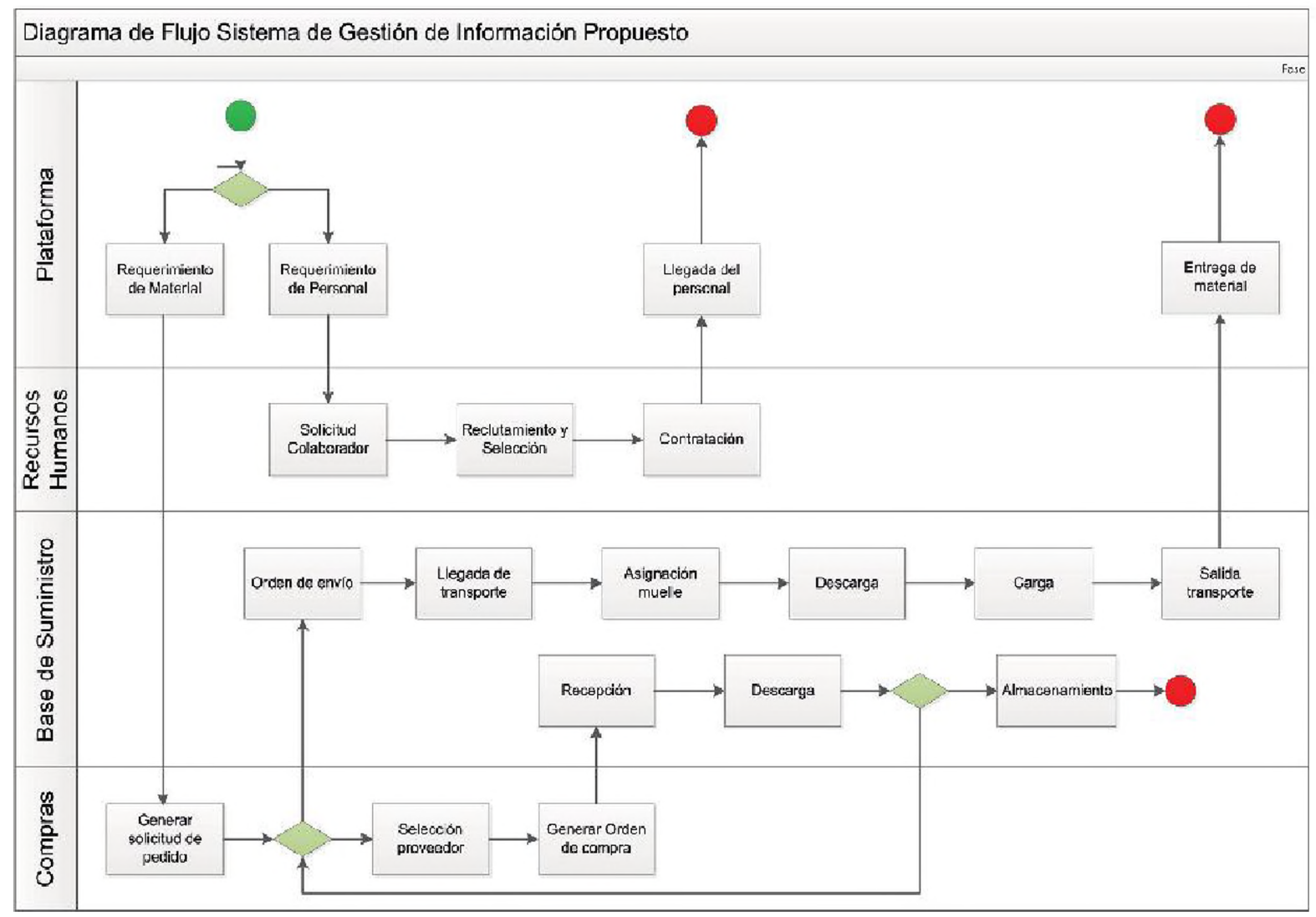

Fuente: elaboración propia.

En este punto, en el que se han expuesto los diagramas de flujo para la respuesta a requeri- mientos de la plataforma; es importante mencionar que en la actualidad y específicamente 
para el caso de estudio, (la perforación off shore en Colombia) existe una debilidad marcada en cuanto al manejo de proveedores. A través de las entrevistas realizadas y en general gracias al acercamiento a personal con experiencia en la cadena de suministro upstream, se cree que en aquellos requerimientos asociados a compras menores o a cambios inesperados en el plan de proyecto para la perforación, la selección de proveedores se realiza de manera intuitiva y sin parámetros unificados de calificación.
Desde el punto de vista de los investigadores, la selección y evaluación de proveedores constituye una fase del proceso fundamental para asegurar el éxito de la operación. Por otro lado, las relaciones estrechas con los proveedores pueden moldearse para encaminar a la creación de aliados estratégicos; de esta manera, ambas partes (proveedor y cliente) aseguran un mejor y más amplio conocimiento del proceso, lo cual, entre otros beneficios, puede resultar en la mitigación de las fallas más frecuentes tanto en el flujo de bienes como en el flujo de información y financiero.

Figura 5. Diagrama de flujo Sistema de Gestión de Información

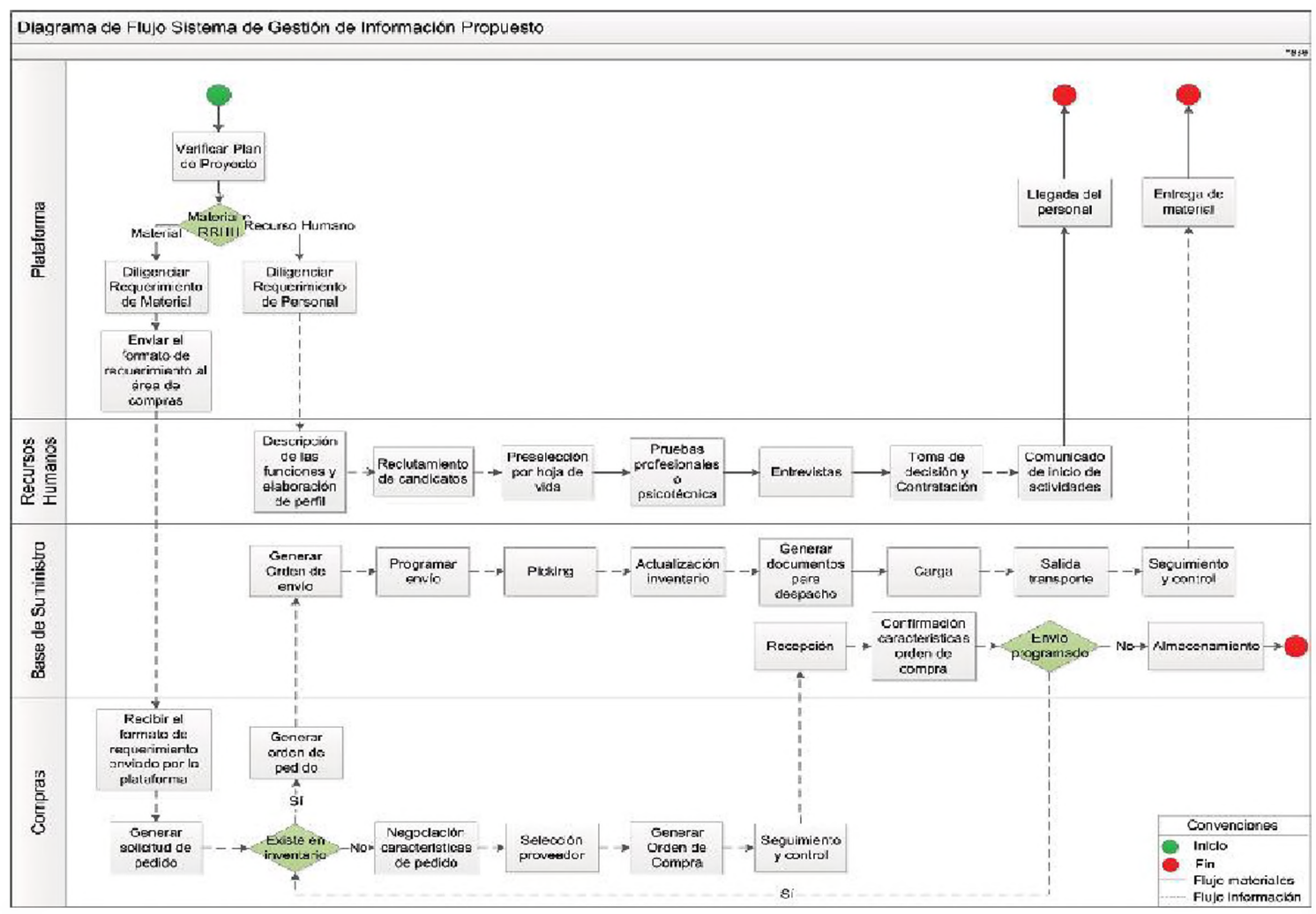

Fuente: elaboración propia.

Desarrollo de la aplicación para el intercambio de información. Para el desarrollo de esta herramienta, se definió Microsoft Access como plataforma tecnológica a utilizar, ya que es un sistema de gestión de bases de datos relacionales, incluida en el paquete Office de Microsoft que permite una interacción rápida bajo una interfaz moderna y sencilla. Se decide tra- 
bajar con bases de datos ya que estas permiten ordenar y organizar información según criterio y con una finalidad concreta; es decir, es un conjunto de datos con características similares, que se distribuyen siguiendo una estructura lógica y persiguen un determinado fin (Varela, 2013).

La herramienta diseñada consta de diferentes módulos que responden a los actores que integran la cadena de suministro y el flujo detallado que se presenta en la Figura 5; empleado(s), proveedor(es), material(es), plataforma y base de suministro o shorebase, los cuales a través de formularios interactúan entre sí en orden de responder a la solicitud de un pedido generado por la plataforma costa afuera. Para lo anterior, los formularios cuentan con un formato en el que se especifican las solicitudes por un responsable, así como botones de envío y registro que permiten comunicar a los interesados y contar con un comprobante del mismo, de igual manera, cuenta con formularios de adición de actores integradores de la cadena como proveedores y empleados y recursos como materiales; formularios de consulta y borrado de registros e informes o plantillas de reporte que proporcionen información de uso general e indicadores.

El funcionamiento del sistema parte de la definición del plan de proyecto que constituye el punto de partida de la operación de perforación; en el que se crean registros de material, de proveedores que los suministren, y los campos de los empleados que serán responsables de los requerimientos.

Dado lo anterior, la plataforma puede empezar a solicitar materiales en orden de sus cronogramas, para ello completa un formato de pedido que posteriormente dirige a compras —división materiales. Posteriormente, materiales verifica la existencia del requerimiento y emite la orden a compras - división proveedores-quién seleccionará el proveedor adecuado para cumplir el requerimiento; una vez seleccionado, gestiona una orden de compra que comparte con la plataforma, con el shorebase y con el proveedor.
El objetivo de compartirlo con plataforma es que ésta conozca el estado de su pedido y quién suministrará su material, para ello recibe un correo y a su vez puede validar el sistema de registro en la opción consulta. La comunicación con shorebase se hace para que éste programe sus instalaciones y transporte en orden de recibir la solicitud para finalmente permitir el ingreso una vez llegue a puerto.

La comunicación al proveedor es el comprobante de la compra y el punto de partida para que este gestione la requisición. Una vez el proveedor dispone lo solicitado en el shorebase, él programa su despacho, para lo cual envía comunicación a compras, y de esta manera da a conocer el cumplimiento de lo solicitado, y a la plataforma para que conozca el estado de su requerimiento. El estado es actualizado continuamente hasta que el transporte retorne al shorebase, en aras que la plataforma pueda prever y controlar sus procesos frente al abastecimiento de su requerimiento.

Al ser la herramienta un modelo de base de datos, la información se puede registrar, almacenar y actualizar a partir de perfiles definidos para tales fines; estos perfiles se diseñaron con el objetivo de garantizar independencia, confidencialidad, confiabilidad y seguridad en los procesos.

El modelo de programación es intuitivo y permite interactuar de forma sencilla con los formularios, adicionalmente cuenta con un diseño cálido que facilita su ubicación y localización de comandos dentro del mismo. Al ser una división de Microsoft, algunos controles son ampliamente conocidos por los usuarios.

\section{Comentarios}

La correcta definición de los actores de la cadena de suministro es indispensable para llevar a cabo una operación logística exitosa, pues a partir de esto se establecen parámetros de in- 
tegración, confidencialidad y flujos óptimos de materiales e información.

La integración de los actores de la cadena de suministro es indispensable para que se pueda definir una red de flujo de información óptima, en la que a través de sus nodos, la información mantenga su valor y calidad, además de permitir su almacenamiento y registro.

Los sistemas de gestión de información ayudan al mejoramiento continuo de las operaciones logísticas siempre y cuando estén definidos bajo parámetros de integración, al permitir administrar la información que fluye a través de ellos y satisfacer los usuarios oportunamente sin riesgo de saturación del mismo, al contar con posibilidades de programación y accesos que permitan controlarla desde su obtención hasta su disposición.

La herramienta diseñada en Microsoft Access cumple con los parámetros de integración de actores de la cadena de suministro, flujo de información a través de nodos funcionales en la operación, calidad, confidencialidad y seguridad de la información a través de la posibilidad de registro y almacenamiento ofrecido por la programación en una plataforma de base de datos.

\section{Referencias}

Ballou, R. (2004). Logística: administración de la cadena de suministro (5ª Ed). México D.F., México: Pearson.

Chima, C. (2007). Supply Chain Management Issues in the Oil and Gas Industries. Journal of Business \& Economics Research, 5, (6),27-36.

Favilla, J., Claessens, D., Mello, U y Flach, B. (2012). Achieving Excellence in Offshore Logistics. Society of petroleum Engineers, 27-29.

Fontana, C., Papa, F., Marte, C., Yoshioka, L., y Sakurai, C. (2014). Intelligent Transportation System as a part of Seaport Terminal Management System. International Journal of Systems Applications, Engineering \& Development, 8, 41-46.

Gutiérrez, H. y De La Vara, R. (2013). Control estadístico de la calidad y Seis Sigma. (2a Ed). México D.F., México: Mc Graw Hill.

Karalkova, A y Kilcheuskaya, K. (2011). X2X: A new conceptual solution on the edge of information systems and supply chain management. Molde University College, Molde, Noruega.

Kukareko y Kiril. (2013). Virtual warehousing in offshore oil and gas platforms' supply chain. Hogskolen i Molde-Vitenskapelig hogskole logistik k.

Porter, M. E. (1985). The Competitive Advantage: Creating and Sustaining Superior Performance. NY: Free Press.

Rajoo, B. (2006). Development of the Kwanda Base Deepwater Logistics Shorebase. Offshore Tecnology Conference, 1-4, May, Houston, Texas, USA.

Varela, C. (2003). Microsoft Access 2003. Nociones básicas. Introducción, tablas y consultas en Microsoft Access. ( $\left.1^{\mathrm{a}} \mathrm{Ed}\right)$. España: Ideas propias Editorial. 